\title{
New metallophamaceutic reduced renal injury induced by non-steroidal anti-inflammatory ${ }^{1}$
}

Clóvis Ney Pinheiro Macêdo' (D), Francisco Evanilso Silva Braga" (D), Ana Paula Bomfim Soares Campelo"II (D), Gabriel Maia Diniz" (D), Luiz Gonzaga de França Lopes ${ }^{\text {IV }}$ (D), Marcos Kubrusly (D), Marcio Wilker Soares Campelov iD

\footnotetext{
' Fellow Master degree, Postgraduate Program in Minimally Invasive Technology and Health Simulation Area, Medical School, Centro Universitário Christus (UNICHRISTUS), Fortaleza-CE, Brazil. Conception and design of the study, technical procedures, acquisition and interpretation of data, manuscript preparation, critical revision.

"Graduate student, Medical School, UNICHRISTUS, Fortaleza-CE, Brazil. Technical procedures, acquisition of data, manuscript preparation.

IIIPhD, Department of Surgery, Universidade Federal do Ceará (UFC), Fortaleza-CE, Brazil. Statistical analysis, manuscript preparation, critical revision.

IVPhD, Full Professor, Chemistry Department, UFC, Fortaleza-CE, Brazil. Conception and design of the study, ruthenium synthese, manuscript preparation, critical revision.

$\checkmark$ PhD, Full Professor, Medical School, UNICHRISTUS, Fortaleza-CE, Brazil. Conception and design of the study, acquisition and interpretation of data, manuscript preparation, critical revision.
}

\begin{abstract}
Purpose: To evaluate the effect of Rut-bpy (Cis-[Ru(bpy)2(SO3)(NO)]PF 6), a novel nitric oxide donor, able to modulate the histological changes caused by the NASID (meloxicam).

Methods: Wistar rats were assigned into three groups ( $n=6$ rats/group): Sham group (saline solution), NSAID group (meloxicam - $15 \mathrm{mg} / \mathrm{kg}$ ) and Rut-bpy group $(100 \mathrm{mg} / \mathrm{kg}$ of Rut-bpy associated with $15 \mathrm{mg} / \mathrm{kg}$ of meloxicam). At the end of experiments, kidneys were removed for histological study, fractal dimension and lacunarity in all animals.

Results: At the histological examination, all animals (six animals - $100 \%$ ) in the NSAID group had membrane thickening and other changes (necrosis, acute tubular congestion and vascular congestion); on the other hand, only one animal (16.6\%) of the Rut-bpy group had congestion. The fractal dimension and lacunarity were greater in the control and Rut-bpy group than in NSAIDs group $(p<0.05)$.
\end{abstract}

Conclusion: Rut-bpy may prevent renal histological changes in rats caused by meloxicam.

Key words: Anti-Inflammatory Agents, Non-Steroidal. Kidney. Ruthenium. Rats. 


\section{- Introduction}

Non-steroidal anti-inflammatory drugs (NSAIDs) are commonly prescribed for the treatment of pain, inflammation and fever. On the other hand, NSAIDs can cause acute renal toxicity, which requires specialist review, renal biopsy, high-dose corticosteroid and/or immunosuppressant treatments, and will normally progress in chronic kidney disease (CKD) $)^{1,2}$.

In this context, many studies demonstrated that nitric oxide (NO) modulation and its isoforms can change the renal diseases evolution ${ }^{3,4}$. NO is soluble mediator produced by several cell types, including renal endothelial. The synthesis is catalyzed by NO synthase (NOS) with the presence of nicotinamide adenine dinucleotide phosphate (NADPH) and oxygen $\left(\mathrm{O}_{2}\right)^{5}$. In this reaction, one of the guanidine nitrogen atoms of $\mathrm{L}$-arginine is oxidized resulting in $\mathrm{N}$-hydroxy-L-arginine. Then, this product is transformed into L-citrulline and $\mathrm{NO}$ as a final product ${ }^{5}$. Hence, the messenger-NO is physiologically synthesized in the kidneys, exerting vital functions in the blood flow homeostasis and renal excretion ${ }^{3,6}$.

Currently, a metallopharmaceutical named Rut-bpy (Cis-[Ru(bpy) 2 (SO3)(NO) $\mathrm{PF}_{6}$ ) can release $\mathrm{NO}$ in vitro and in vivo, as well as it can reduce inflammatory processes through NF-kB inhibition ${ }^{7,8}$.

Considering the lack of studies associating NSAIDs with a NO-donor to prevent renal lesions, in this study we aimed to compare the prolonged use of NSAID alone and the use of NSAIDs together with new metallopharmaceutical NO donor. For this purpose, we studied: (1) the effects of meloxicam (a kind of NSAIDs) on renal histology in rat; (2) and the capacity of new metallapharmaceutical to attenuate meloxican-induced renal injury.

\section{- Methods}

All procedures and animal handling were conducted in accordance with the Guide for the Care and Use of Laboratory Animals from the Brazilian College of Animal Experimentation, after approval by the local ethics committee (protocol \#62). The study was designed to minimize the number of animals required for the experiments.

Eighteen male Wistar rats weighing 200-230 g were used and maintained in the Laboratory of Experimental Surgery - LABCEX (UNICHRISTUS). Food and water were available ad libitum and the animals were maintained in the same environmental conditions in individual cages during a $12 \mathrm{~h} / 12 \mathrm{~h}$ light/dark cycle.

\section{New metallopharmaceutical (Rut-bpy)}

The Rut-bpy (cis-[Ru(bpy)2(SO3)(NO)]PF6) was synthesized and purified at the Department of Organic and Inorganic Chemistry, Universidade Federal do Ceará (Brazil), following procedures described elsewhere ${ }^{9}$. Rut-bpy (working solution of $1.95 \mathrm{mM}$ ) was administered intraperitoneally at a dose of $0.15 \mathrm{mmol} / \mathrm{kg}$ (equivalent to $100 \mathrm{mg} / \mathrm{kg}$ ). This dosage was based on previous experiments, which showed low toxicity to rats ${ }^{10}$ and benefits in others experimental model in vivo ${ }^{11}$.

\section{Experimental design}

Animals were randomly divided into three groups of six animals each as follows:

a. Control group (control): saline solution administration (vehicle) intraperitoneally injected for 10 days.

b. NAIDs group (NSAID): meloxicam intramuscular administration (15 mg/kg), daily for 10 days.

c. Rut-bpy group (NSAID+Ru): Rut-bpy administration (100 mg/kg), intraperitoneally injected associated with meloxicam (15 mg/kg - intramuscular) for 10 days.

\section{Histopathology}

At the end of the experiments (tenth day) all animals were killed by an overdose of anesthetics (ketamine + xylazine). Renal tissue samples were collected from all animals. Tissue samples were fixed in formalin for 24 hours and transferred to $70 \%$ ethanol solution. Further processing included paraffin embedding and sectioning to generate 5 - $\mu \mathrm{m}$-thick tissue coronal sections to be mounted on glass slides. The slides were stained using hematoxylin and eosin and assessed by a pathologist (RP) following procedures described elsewhere ${ }^{12}$.

\section{Analysis of fractal geometry (fractal dimension and lacunarity)}

The fractal dimension (DF) and lacunarity (LAC) were calculated using box counting method ${ }^{13,14}$ with the aid of free image analyzer program IMAGE-J ${ }^{\circ}$ and the public domain FracLac plug-in (http://rsbweb.nih.gov/ij/).

During the preparation to avoid selection bias, in the present study it was chosen the option to analyze the entire histological renal image and for the analysis the TIFF image was transformed in binary automatically by the software, so it was transformed into an image consisting of black pixels (intensity 0) on white background (intensity 255). 
The size of the box was programmed to increase progressively (box $=2,3,4,8,16,32,64$ ). The data obtained were automatically organized in spreadsheets and electronic graphics by Image- $J^{\circ}$.

Fractal dimension and lacunarity were evaluated using the automated block counting method, without interference from the researchers, and a binary image representing the object analyzed was divided into a series with several L-side squares. Next, the number $\mathrm{N}$ (L) of sheets containing the object (s) of interest were counted. The value of $L$ varied exponentially, i.e., using $L=1,2,4,8 \ldots .$. to the limit of the image dimensions. The number that followed was calculated like this: once between the scale of the part of the images of the number of the plot and the digital number of images ${ }^{15}$.

The DF corresponds to the alpha angular coefficient of the modulus-adjusted straight line and is defined as the slope of the straight line in the graph that correlates the degree of occupation of the space $N(L)$ with the variable dimensional scales $L$ to which it is analyzed. It reveals the level of regularity of an object between the different spatial scales (BOUDA $M, 2016)$, being estimated by the radius of log change $N(L)$ by the log change of scale $\mathrm{L}$ and having the following equation: $\mathrm{DF}=$ variation $\log N(\mathrm{~L}) /$ variation $\log \mathrm{L}^{15}$.

\section{Statistical analysis}

Data were expressed as mean \pm SD. After a Kolmogorov-Smirnov test of normality, parametric data were submitted to one-way analysis of variance followed by Tukey's multiple comparison test. Histopathological data were analyzed with chisquare test. The level of statistical significance was set at $5 \%$.

\section{- Results}

\section{Rut-bpy attenuated NSAID-induced histopathology, fractal dimension and lacunarity}

Histopathological examination of the renal tissue sections revealed that all rats (six animal - 100\%) from meloxcam group ( $N=6)$ (NSAID) had necrosis, acute tubular congestion, vascular congestion, thickening of tubular membranes. In the group NSAID+Ru $(\mathrm{N}=6)$, only 1 animal $(16.6 \%)$ presented a discrete eosinophilic infiltrate and mild vascular congestion; the other animals (five animals) had no histopathological alterations (Fig. 1). In the control group $(N=6)$ there was no animals with renal injury. The analysis of the discrepancy measurement between the observed and the expected frequencies (chi-square test) between the groups showed a significant difference between the groups NSAID vs. NSAID + Ru (chi-square = 14.49; $p<0.05$ ); there was no statistically significant difference between NSAID + Ru vs. Control ( $p>0.05)$.
A

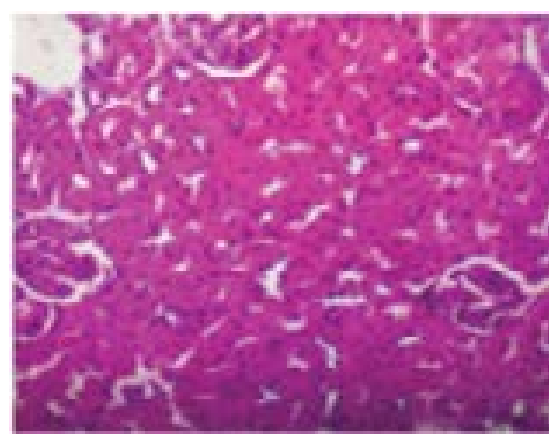

B
C

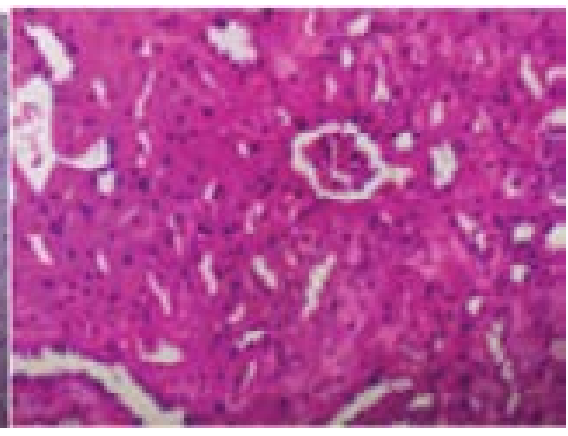

Figure 1 - Representative images of the renal tissue histopathology of the Control, NSAID and NSAID+Ru groups. A) Normal slide of glomeruli and renal tubules of the Control group. B) Renal tissue of animals that received meloxicam, showed tubular congestion, thickening of membranes. C) Renal tissue of animals receiving meloxicam+Rut-bpy (group $\mathrm{NASID}+\mathrm{Ru}$ ) showed normal renal tubules and glomeruli. Image magnification $\times 200$. Abbreviations: NSAID=meloxicam; $\mathrm{Ru}=$ nitrosil ruthenium (Rut-bpy).

The fractal dimension in the control and NSAID+Ru groups did not differ significantly ( $p>0.05$ ). However, we found differences between control versus NSAID and
NSAID versus NSAID+Ru, $(p<0.05)$. The Fractal dimension was greater in the control and NSAID+Ru group than in the group receiving NSAID alone (Fig. 2). 


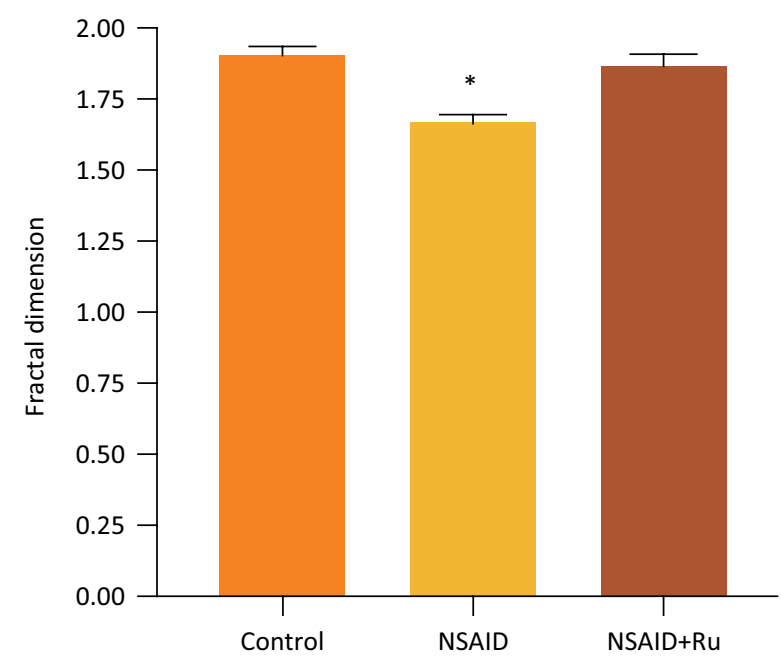

Figure 2 - Rut-bpy administration associated with meloxicam. Fractal dimension analysis of the images of the renal tissue histopathology from six rats in each group. Values are expressed as mean \pm SD. ${ }^{*} p<0.05$, as compared groups: NSAID vs. Control or NSAID vs. NSAID+Ru. Abbreviations: NSAID=meloxicam; $\mathrm{Ru}=$ nitrosil ruthenium (Rut-bpy).

The lacunarity was significantly smaller in the NSAID group as compared with the NSAID+Ru group $(p<0.05)$. The Control and NSAID+Ru groups did not differ significantly ( $p>0.05$ ) (Fig. 3).

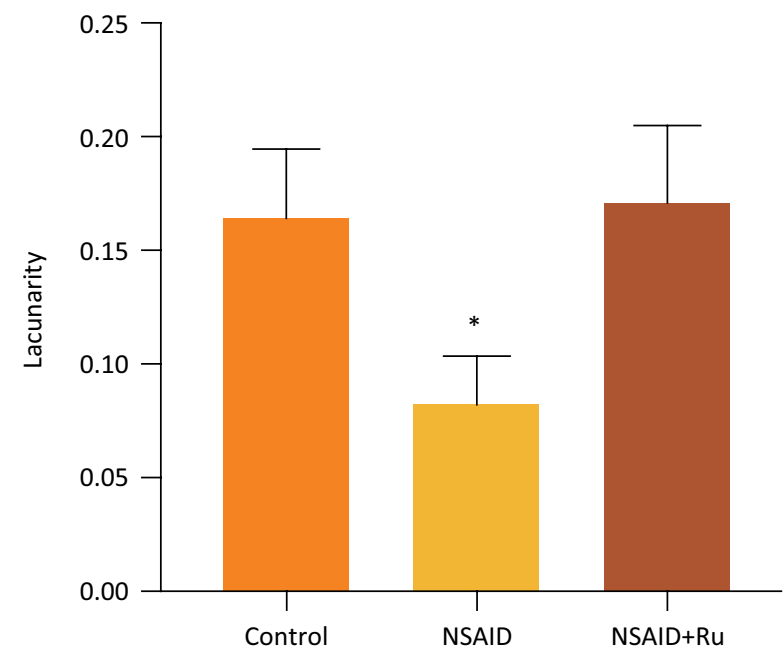

Figure 3 - The lacunarity of the renal images from six rats in each group. Results are expressed as mean \pm SD. ${ }^{*} p<0.05$, as compared groups: control vs. NSAID or NSAID vs. NSAID+Ru. Abbreviations: NSAID=meloxicam; $\mathrm{Ru}=$ nitrosil ruthenium (Rut-bpy).

\section{- Discussion}

Renal diseases can be associated with the use of several drugs, especially NSAIDs, leading to acute interstitial glomerulonephritis, which can become chronic and are also called papillary necrosis ${ }^{16,17}$.

NSAIDs are one of the most prescribed drugs in the medical practice, especially to alleviate migraine, pain after surgery, arthritis, and dysmenorrhea due to their analgesic and anti-inflammatory effects in a wide range of clinical situations.

However, the use of NSAIDs should be restricted due to its adverse effects in the gastrointestinal tract and renal toxicity, which can be life-threatening due to acute renal toxicity.

Furthermore, nephrotic syndrome, a disorder caused by glomerulonephritis, diabetes, aminoglycosides, and indiscriminate use of NSAIDs, does not have an excellent therapeutic approach so far and its physiopathology lacks solid mechanisms.

The studies in vitro in renal tubular cell culture showed that immunoinflammatory disorder was associated to renal injury, with increased production of monocytes, MCP-1, endothelin-1, RANTES and NFkB ${ }^{18,19}$.

In rats with proteinuria, inhibition of $N F k B$ is able to protect them from nephritis ${ }^{20,21}$. NFKB activated in podocytes. NFkB activation and NO production by NOSi are known to lead to cell damage, as shown in experimental rat model $^{8}$, and also promote glomerulonephritis ${ }^{22}$.

NF-kB activation plays a dual role in cell death and survival, depending on cell type, developmental stage and apoptotic stimuli ${ }^{23}$. The NO donors in experimental model have shown that they can inhibit NFkB activation and may prevent cellular damage caused by both activation of inflammatory factors and activation or inactivation of various protein kinases ${ }^{24-26}$.

The NO donors, when applied in biological systems, release NO and are able to mimic the endogenous NO release response or replace an endogenous NO deficiency ${ }^{27,28}$. Recently, many metallodrugs have been studied such as NO-donors or NO-scavengers, including ruthenium nitrosyl complexes ${ }^{29}$.

New compounds obtained from ruthenium nitrosyl complexes and synthesized in the laboratory of the Department of Inorganic and Organic Chemistry, UFC (Cis-[Ru(bpy)2(SO3)(NO)]PF6, Ru-Bpy) according to the technique described by Silva et. al. ${ }^{9}$ are water soluble and remain stable when exposed to the environment. These drugs can release $\mathrm{NO}$ and inhibit NFk-B in vivo ${ }^{8}$ and in vitro ${ }^{7,30}$.

In this study, we used a new NO-donor metallopharmaceutic (Rut-bpy) simultaneously 
administered with meloxicam. This is the first report that associates a NO-donor and an NSAID with the potential to prevent pathologic modification of histopatologic and biochemistry in rat's kidney.

In renal disease associated with the use of nonsteroidal anti-inflammatory drugs (NSAIDs), histological changes often occur with predominantly lymphocyte infiltrate in the interstitium, and vacuolar degeneration of the proximal and distal tubules ${ }^{17,31}$.

In this study, Wistar rats that received exclusively meloxicam showed renal histological changes (basal membrane thickening and tubulointerstitial nephritis), which are in agreement with other studies ${ }^{32-34}$. On the other hand, rats that received meloxicam associated with nitrosyl ruthenium did not present such histological alterations and this is our main result. The histologic exam was performed by a blindly experienced pathologist.

These histological results support that the use of meloxicam associated with nitrosyl ruthenium may qualitatively reduce meloxicam-induced renal damage. Rut-bpy probably protects renal cells by nitric oxide (NO) donation at the vascular endothelium level, promoting renal protection when administrated in combination with meloxicam.

Other analyses that were performed with the histologic slides were: automatic dimension measures (fractal dimension and lacunarity) without human interference, which are able to determine details with the magnification of the image resolution and represent an estimate of the structural complexity of the tissue ${ }^{35}$.

Fractal dimension (DF) is a quantifier of the geometric complexity of an object or image. It is able to do description and measurement of the self-similarity of the analyzed object, enables the comparison of parts of the structure with the structure as a whole ${ }^{35-39}$. Fractal geometry has no units in the international system of unit, because it is a ratio between logarithms variation of the same unit.

Several studies have evaluated fractal dimensioning of histological slides to identify histological changes in several experiments, for example: assessing the degree of tumor invasiveness by quantifying the fractal dimension of the malignant epithelium/ stroma interface ${ }^{40}$, liver tissue with different degrees of cirrhosis due to hepatitis $\mathrm{C}^{41}$, identification of dysplasia of lesions in oral cavity epithelium ${ }^{42}$, in a study of quantification of the degree of myocardial cell rejection ${ }^{43}$, estimation of nephron integrity ${ }^{14}$ and study pointed out that the vascular networks indirectly reflecting the architecture of stromal frameworks might have measurable and understandable differences between renal oncocytomas and ChRCCs (chromophobe renal cell carcinomas) ${ }^{44}$.

Interestingly, the animal that received only meloxicam had a smaller fractal dimension than the control, probably due to nephritis caused by NSAID. This fact is congruent with the histological alterations found, since it had smaller fractal dimension and smaller similarity between others groups.

On the other hand, animals that received meloxicam and ruthenium simultaneously presented similar renal cytoarchitecture with the control group, corroborating the histological findings. In addition, we improved our result of fractal dimension evaluating other fractal parameter - the lacunarity.

The term lacunarity was introduced by Mandelbrot ${ }^{39}$ to describe features that can differentiate objects by varying the spatial distribution and the size of voids (gaps) that exist in a given object or image. Thus, a lacunarity is an element of fractal geometry that serves to describe the texture of an image $e^{45}$.

A low lacunarity fractal object or image has been more homogeneous because it has, in probabilistic terms, the same size as the gaps, being the true inverse ${ }^{46}$.

The tubular congestion, edema and thickening of membranes may reduce the gaps in the renal tissue, this spatial distribution of cytoarchitecture (gaps) may have been confirmed by the results of lacunarity which showed smaller in meloxican group than the other groups in study.

Fractal analysis, mainly through its fractal dimension and lacunarity parameters, has been increasingly used in the evaluation of images and inferences about the anatomical structure from the structural and architectural point of view ${ }^{47}$.

This is the first time that renal tissues affected with nephrophatology by the use of meloxicam are under study with fractal dimension and lacunarity. Though there were many ways to measure the fractal dimension, we chose the "box-counting" method for both its simplicity and suitability of application for the objects seen in the histological sections.

\section{- Conclusions}

The metallodrug tested may play an important role in the prevention of NSAIDs-induced renal lesions. However, other studies are necessary to further investigate the ruthenium function and role in this pathology, including the evaluation of other renal markers. This drug may therefore be considered an attractive candidate for upcoming investigations with experimental renal disease by NSAID. 


\section{- References}

1. Leonard CE, Freeman CP, Newcomb CW, Reese PP, Herlim M, Bilker WB, Hennessy S, Strom BL. Proton pump inhibitors and traditional nonsteroidal anti-inflammatory drugs and the risk of acute interstitial nephritis and acute kidney injury. Pharmacoepidemiol Drug Safety. 2012;21(11):1155-72. doi: 10.1002/pds.3329.

2. Cabassi A, Tedeschi S, Perlini S, Verzicco I, Volpi R, Gonzi G, Canale SD. Non-steroidal anti-inflammatory drug effects on renal and cardiovascular function: from physiology to clinical practice. Eur J Prev Cardiol. 2019;14:2047487319848105. doi: $10.1177 / 2047487319848105$.

3. Fry BC, Edwards A, Layton AT. Impact of nitric-oxidemediated vasodilation and oxidative stress on renal medullary oxygenation: a modeling study. Am J Physiol Renal Physiol. 2016;310(3):237-47. doi: 10.1152/ ajprenal.00334.2015.

4. Abdellatif KR, Abdelall EK, Bakr RB. nitric oxide-nasids donor prodrugs as hybrid safe anti-inflammatory agents. Curr Top Med Chem. 2017;17(8):941-55. doi: 10.2174/156 8026616666160927153435.

5. Mónica FZ, Bian K, Murad F. The endothelium-dependent nitric oxide-cGMP pathway. Adv Pharmacol. 2016;77:1-27. doi: 10.1016/bs.apha.2016.05.001.

6. Ahmad A, Dempsey SK, Daneva Z, Azam M, Li N, Li PL, Ritter JK. Role of nitric oxide in the cardiovascular and renal systems. Int J Mol Sci. 2018;19(9). pii: E2605. doi: 10.3390/ijms19092605.

7. Cerqueira JB, Silva LF, Lopes LG, Moraes ME, Nascimento NR. Relaxation of rabbit corpus cavernosum smooth muscle and aortic vascular endothelium inducedby new nitric oxide donor substances of the nitrosylruthenium complex. Int Braz J Urol. 2008; 34(5):638-45. doi: $10.1590 / s 1677-55382008000500013$.

8. Campelo MW, Oriá RB, Lopes LG, Brito GA, Santos AA, Vasconcelos RC, Silva FO, Nobrega BN, Bento-Silva MT, Vasconcelos PR. Preconditioning with a novel metallopharmaceutical NO donor in anesthetized rats subjected to brain ischemia/reperfusion. Neurochem Res. 2012;37(4):749-58. doi: 10.1007/s11064-011-0669-x.

9. Silva FON, Araujo SXB, Holanda AKM, Meyer E, Sales FAM, Diogenes ICN, Carvalho IMM, Moreira IS, Lopes LGF. Synthesis, characterization, and NO release study of the cis- and trans-[Ru(Bpy)2(SO3)(NO)] complexes. Eur J Inorg Chem. 2006;2006(10):2020-6. doi: 10.1002/ ejic. 200500871.

10. Silva JJN, Guedes PMM, Zottis A, Balliano TL, Silva FON, Lopes LGF, Ellena J, Oliva G, Andricopulo AD, Franco DW, Silva JS. Novel ruthenium complexes as potential drugs for Chagas's disease: enzyme inhibition and in vitro/in vivo try- panocidal activity. Br J Pharmacol. 2010;160:260-9. doi: 10.1111/j.1476-5381.2009.00524.x.

11. Fricker SP, Slade E, Powell NA, Vaughan OJ, Henderson GR, Murrer BA, Megson IL, Bisland SK, Flitney FW. Ruthenium complexes as nitric oxide scavengers: a potential therapeutic approach to nitric oxide- mediated diseases. Br J Pharmacol. 1997;122:1441-9. doi: 10.1038/ sj.bjp.0701504.
12. Putra A, Pertiwi D, Milla MN, Indrayani UD, Jannah D, Sahariyani M, Trisnadi S, Wibowo JW. Hypoxiapreconditioned MSCs have superior effect in ameliorating renal function on acute renal failure animal model. Open Access Maced J Med Sci. 20190;7(3):305-10. doi: 10.3889/ oamjms.2019.049.

13. Smith TG Jr, Lange GD, Marks WB. Fractal methods and results is cellular morphology-dimensions, lacunarity and multifractals. J Neurosci Methods. 1996;69(2):123-36. doi: 10.1016/S0165-0270(96)00080-5.

14. Nigro $M$, Viggiano $D$, Ragone $V$, Trabace $T$, di Palma A, Rossini M, Capasso G, Gesualdo L, Giogliotti G. A cross-sectional study on the relationship between hamtological data and quantitative morphological índices from kidney biopsis in diferent glomerular diseas. BMC Nephrol. 2018;19(1):62. doi: 10.1186/ s12882-018-0846-0.

15. Bouda M, Caplan JS, Saiers JE. Box-counting dimension revisited: presenting an efficient method of minimizing quantization error and an assessment of the self-similarity of structural root systems. Front Plant Sci. 2016;18(7):149. doi: 10.3389/fpls.2016.00149.

16. Zhang X, Donnan PT, Bell S, Guthrie B. Non-steroidal antiinflammatory drug induced acute kidney injury in the community dwelling general population and people with chronic kidney disease: systematic review and metaanalysis. BMC Nephrol. 2017;18(1):256. doi: 10.1186/ s12882-017-0673-8.

17. Wu H, Huang J. Drug-induced nephrotoxicity: pathogenic mechanisms, biomarkers and prevention strategies. Curr Drug Metab. 2018;19(7):559-67. doi: 10.2174/138920021 8666171108154419.

18. Wang Y, Chen J, Chen L, Tay YC, Harris DC. Induction of monocyte chemoattractant protein-1 in proximal tubule cells by urinary proteins. J Am Soc Nephrol. 1997; 8:1537-45. doi: 1046-6673/08010-1537\$03.00/0.

19. Devocelle A, Lecru L, François $H$, Desterke C, Gallerne $C$, Eid $P$, Estelle $O$, Azzarone B, Giron-Michel J. Inhibition of TGF-61 signaling by IL-15: a novel role for IL15 in the control of renal epithelial-mesenchymal transition: IL-15 counteracts TGF-81-induced EMT in renal fibrosis. Int J Cell Biol. 2019;2019:9151394. doi: 10.1155/2019/9151394.

20. Rangan GK, Wang Y, Tay YC, Harris DC. Inhibition of NFkB activation reduces cortical tubule interstitial injury in proteinuric rats. Kid Int. 1999;6:116-34. doi: 10.1046/j.1523-1755.1999.00529.x.

21. Camici M. The nephrotic syndrome is an immunoinflammatory disorder. Med Hypotheses. 2007;68(4):900-5. doi: 10.1016/j.mehy.2006.04.072.

22. Ni Z, Vaziri ND. Downregulation of nitric oxide synthase in nephrotic syndrome: role of proteinuria. Biochim Biophys Acta. 2003;1638(2):129-37. doi: 10.1016/s09254439(03)00061-9.

23. Kaltschmidt B, Heinrich $M$, Kaltschmidt C. Stimulusdependent activation of NF-kappaB specifies apoptosis or neuroprotection in cerebellar granule cells. Neuromolecular Med. 2002;2:299-309. doi: 10.1385/ NMM:2:3:299. 
24. Coert BA, Anderson RE, Meyer FB. A comparative study of the effects of two nitric oxide synthase inhibitors and two nitric oxide donors on temporary focal cerebral ischemia in the Wistar rat. J Neurosurg. 1999;90(2):332-8. doi: 10.3171/jns.1999.90.2.0332.

25. Arandarcikaite O, Jokubka R, Borutaite V. Neuroprotective effects of nitric oxide donor NOC-18 against brain ischemia-induced mitochondrial damages: role of PKG and PKC. Neurosci Lett. 2015;586:65-70. doi: 10.1016/j. neulet.2014.09.012.

26. Wainwright MS, Grundhoefer D, Sharma S, Black SM. A nitric oxide donor reduces brain injury and enhances recovery of cerebral blood flow afterhypoxia-ischemia in the newborn rat. Eurosci Lett. 2007;415(2):124-9. doi: 10.1016/j.neulet.2007.01.019.

27. Ignarro LJ, Cirino G, Casini A, Napoli C. Nitric oxide as a signaling molecule in the vascular system: an overview. J Cardiovasc Pharmacol. 1999;34:879-86. doi: 10.1097/00005344-199912000-00016.

28. Ignarro LJ, Napoli C, Loscalzo J. Nitric oxide donors and cardiovascular agents modulating the bioactivity of nitric oxide: an overview. Circ Res. 2002;90:21-8. doi: 10.1161/ hh0102.102330.

29. Lunardi CN, Da Silva RS, Bendhack LM. New nitric oxide donors based on ruthenium complexes. Braz J Med Biol Res. 2009;42:87-93. doi: 10.1590/s0100$879 \times 2009000100013$.

30. Wieraszko A, Clarke MJ, Lang DR, Lopes LG, Franco DW. The influence of NO-containing ruthenium complexes on mouse hippocampal evoked potentials in vitro. Life Sci. 2001;68(13):1535-44. doi: 10.1016/s00243205(01)00951-1.

31. Harirforoosh S, Asghar W, Jamali F. Adverse effects of nonsteroidal antiinflammatory drugs: an update of gastrointestinal, cardiovascular and renal complications. J Pharm Pharm Sci. 2013;16(5):821-47. doi: 10.18433/ j3vw2f.

32. Burukoglu D, Baycu C, Taplamacioglu F, Sahin E, Bektur E. Effects of nonsteroidal anti-inflammatory meloxicam on stomach, kidney, and liver of rats. Toxicol Ind Health. 2016;32(6):980-6. doi: 10.1177/0748233714538484.

33. Asghar W, Aghazadeh-Habashi A, Jamali F. Cardiovascular effect of inflammation and nonsteroidal anti-inflammatory drugs on renin-angiotensin system in experimental arthritis. Inflammopharmacology. 2017 Apr 7. doi: 10.1007/s10787017-0344-1.

34. Asghar W, Jamali F. The effect of COX-2-selective meloxicam on the myocardial, vascular and renal risks: a systematic review. Inflammopharmacology. 2015;23(1):116. doi: 10.1007/s10787-014-0225-9.
35. Dierick F, Nivard AL, White O Buisseret F. Fractal analyses reveal independent complexity and predictability of gait. PLoS One. 2017;12(11):e0188711. doi: 10.1371/journal. pone.0188711.

36. Canals M, Solís R. Geometry of living systems and its importance in Medicine. Rev Med Chil. 2005;133(9):1097107. doi: S0034-98872005000900015.

37. Losa GA, Nonnenmacher TF. Self-similarity and fractal irregularity in pathologic tissues. Mod Pathol. 1996;9(3):174-82.

38. Cross SS, Start RD, Stephenson TJ, Cotton DW, Variend $\mathrm{S}$, Underwood JC. Fractal geometric analysis of the renal arterial tree in infants and fetuses. Pediatr Pathol Lab Med. 1995;15(2):259-68. doi: 10.3109/15513819509026961.

39. Mandebrolt B. The fractal geometry of nature. New York: WH. Freeman Company; 1983.

40. Bizzarri M, Giuliani A, Cucina A, Anselmi FD, Soto AM, Sonnenschein C. Fractal analysis in a systems biology approach to cancer. Semin Cancer Biol. 2011;21(3):17582. doi: 10.1016/j.semcancer.2011.04.002.

41. Dioguardi N, Grizzi F, Fiamengo B, Russo C. Metrically measuring liver biopsy: A chronic hepatitis B and C computer-aided morphologic description. World J Gastroenterol. 2008;14(48):7335-44. doi: 10.3748/ wjg.14.7335.

42. Abu-Eid R, Landini G. Morphometrical differences between pseudo-epitheliomatous hyperplasia in granular cell tumours and squamous cell carcinomas. Histopathology. 2006;48(4):407-16. doi: 10.1111/j.13652559.2006.02350.x.

43. Moreira RD, Moriel AR, Murta Junior LO, Neves LA, Godoy MF. Fractal dimension in quantifying the degree of myocardial cellular rejection after cardiac transplantation. Rev Bras Cir Cardiovasc. 2011;26(2):155-63. doi: 10.1590/ s0102-76382011000200004.

44. Karslioğlu Y, Günal A, Kurt B, Ongürü O, Ozcan A. Fractal dimension of microvasculature in renal oncocytomas and chromophobe renal cell carcinomas. Pathol Res Pract. 2009;205(10):677-81. doi: 10.1016/j.prp.2009.03.004.

45. Smith TG Jr, Lange GD, Marks WB. Fractal methods and results in cellular morphology dimensions, lacunarity and multifractals. J Neurosci Methods. 1996;69(2):123-36. doi: 10.1016/S0165-0270(96)00080-5.

46. Tolle RC, McJunkin TR, .McJunkin, Rohrbaugh TD, LaViolette RA. Lacunarity definition for ramified data sets based on optimal cover. Physica D: Nonlinear Phenomena. 2003;179:129-52. doi: 10.1016/S0167-2789(03)00029-0.

47. Lopes R, Betrouni N. Fractal and multifractal analysis: a review. Med Image Anal. 2009;13(4):634-49. doi: 10.1016/j.media.2009.05.003. 


\section{Correspondence:}

Marcio Wilker Soares Campelo

Rua João Adolfo Gurgel, 133

60.192-345 Fortaleza - CE Brasil

marciowilker@bol.com.br

Received: Aug 15, 2019

Review: Oct 18, 2019

Accepted: Nov 13, 2019
Conflict of interest: none

Financial sources: UNICHRISTUS, INPEC, and FUNCAP

${ }^{1}$ Research performed at Laboratory of Experimental Surgery (LABCEX), Centro Universitário Christus (UNICHRISTUS), and Scientific Research and Teaching Institute (INPEC), Fortaleza-CE, Brazil. Part of Master degree thesis, Postgraduate Program in Minimally Invasive Technology and Health Simulation Area. Tutor: Prof. Marcio Wilker Soares Campelo.

This is an Open Access article distributed under the terms of the Creative Commons Attribution License, which permits unrestricted use, distribution, and reproduction in any medium, provided the original work is properly cited. 